\title{
Retórica y comunicación en red: convergencias y analogías. Nuevas propuestas docentes ${ }^{1}$
}

\author{
Inmaculada BERLANGA FERNÁNDEZ \\ Universidad Internacional de la Rioja \\ inmaculada.berlanga@unir.net \\ Jordi AlBERICH PASCUAL \\ Universidad de Granada \\ jalberich@ugr.es
}

Recibido: 19/07/2012

Aceptado: 29/10/2012

\section{Resumen}

La docencia en Comunicación precisa una continua actualización de sus fórmulas. Esta investigación propone la introducción de contenidos teóricos y prácticos de la ciencia Retórica en los planes de estudio de aquellos campos que giran en torno a la comunicación, basándose en el informe sobre los Títulos de grado en Comunicación creado por la ANECA y en sus propuestas para los futuros periodistas y comunicadores. El trabajo de campo realizado sobre la red social Facebook arroja unos resultados que permiten entrelazar la Retórica y nuestro presente comunicativo. Un presente mediado por la emergencia de las redes como nuevo paradigma. En un momento histórico como el que vivimos será de gran utilidad recuperar unos parámetros que devuelvan el pensamiento profundo y crítico en favor de una comunicación más humana.

Palabras clave: Comunicación, Retórica, Redes Sociales, Docencia, ANECA

\section{Rethoric and web communication: points of convergence and analogies. New pedagogical proposals}

\begin{abstract}
Education in Communication requires an on-going revision of methods. This paper suggests the introduction of theoretical and practical content of the Rhetoric science in the curricula of communication studies and other studies revolving around communication. This suggestion is based on the ANECA reports for the degree Bachelor of Arts in Communication, together with ANECA proposals for future journalists and communicators. Our fieldwork in the social network Facebook sheds positive results allowing us to interlink Rhetoric and our present communication environment. This present is mediated by social networks, emerging as a new paradigm. Retrieving parameters which devolve deep and critical thinking in favor of a more human communication should be of great use in our historical times.
\end{abstract}

Keywords: Communication, Rhetoric, Social networks, Teaching, ANECA

\section{Referencia normalizada}

BERLANGA FERNÁNDEZ, Inmaculada; ALBERICH PASCUAL, Jordi (2012): "Retórica y comunicación en red: convergencias y analogías. Nuevas propuestas docentes". Estudios sobre el mensaje periodístico. Vol. 18, núm. especial octubre, págs.: 141-150. Madrid, Servicio de Publicaciones de la Universidad Complutense.

Sumario: 1. Introducción. 2. Fuentes y metodología. 3. Comunicación y Retórica; 3.1. Presencia de la retórica en la comunicación en Internet; 3.2. Presencia de la retórica en las redes sociales; 3.3. Análisis retórico de una red: el caso Facebook. 4. Conclusiones: hacia una docencia más original. 5. Referencias bibliográficas

${ }^{1}$ Estudio enmarcado en la Convocatoria de Proyectos I+D del Ministerio de Economía y Competitividad con clave: EDU2010-21395-C03-03, titulado "La enseñanza obligatoria ante la competencia en comunicación audiovisual en un entorno digital". 


\section{Introducción}

La docencia en Comunicación precisa, quizás más que otras áreas, una continua actualización de sus fórmulas. El nuevo contexto socio-cultural creado por la aparición de Internet ha propiciado una cultura red que, si bien se extiende a todos los ámbitos del saber, en los estudios de Comunicación se presenta como el hábitat natural.

El informe sobre los Títulos de grado en Comunicación creado por la Agencia Nacional de Evaluación de la Calidad y la Acreditación Española recoge expresamente que el comunicador requiere una serie de saberes y habilidades interdisciplinares: la formación en Ciencias Sociales y Humanidades para proporcionar una competencia contextual básica de naturaleza transdisciplinar; la formación en las capacidades expresivas y en los lenguajes de cada uno de los medios y formatos de comunicación, o la capacidad de análisis tanto en medios icónicos como en los entornos digitales. Estos conocimientos y habilidades actuarán en el futuro graduado en Comunicación como garante de una adecuada preparación encaminada a su posterior vida laboral (ANECA, 2000: 320-321).

En esta línea, la presente investigación propone la introducción de contenidos teóricos y prácticos de la ciencia Retórica en los planes de estudio de aquellos campos que giran en torno a la comunicación, bien mediante una asignatura específica, bien de forma transversal. Para la fundamentación de esta propuesta abundaremos en la convergencia entre comunicación y Retórica, mostraremos el resultado de un una investigación que analiza la presencia de la Retórica en la comunicación a través Internet y de las redes sociales, y finalmente extraeremos las conclusiones pertinentes.

\section{Metodología}

La base científica de esta propuesta se fundamenta en una investigación sobre el discurso retórico que se produce en las redes sociales on line, concretamente en Facebook, y en sus dos dimensiones: el discurso de la propia plataforma y el discurso que emplean los usuarios de la red y en sus relaciones entre sí. La aplicación de una metodología que combina las técnicas del análisis documental, el análisis de contenido de los muros de distintas microrredes y las entrevistas científicas, arroja unos resultados que permiten entrelazar la Retórica y nuestro presente comunicativo, mediado por las redes, como realidades convergentes y análogas.

A lo largo de la Historia, las tesis oratorias formuladas por los clásicos no han perdido actualidad, inspirando o actuando de soporte a otras teorías más modernas (retórica política, jurídica, periodística, académica, conversacional, etc.). "Especialmente la Retórica ha ejercido una notable influencia en la elaboración de la teoría de la comunicación actual que continua y continuará transitando por vías recientemente abiertas, como las de la hipertextualidad, interactividad o la multimedialidad" (Albaladejo, 2008: 19).

\section{Comunicadores y Retórica}

El campo expandido de la comunicación se enfrenta en la actualidad a una serie de retos en los que se pone a juego incluso la propia identidad histórica de los Media como tales (Tubella y Alberich, 2012: 11). Ante la enorme extensión de las redes por 
todos los ámbitos del saber, los diferentes perfiles del comunicador tienen estas plataformas como su medio vital de trabajo. En el campo de la Información, ante los problemas actuales que rodean a la empresa informativa, los periodistas están obligados a reforzar aquellos conocimientos que incrementan su valor profesional (Salaverría, 2012: web) dirigiendo sus esfuerzos al aprendizaje de profesiones emergentes que se desarrollan en la web 2.0. Si miramos al Entretenimiento, existen datos elocuentes sobre la convergencia del tiempo libre de los ciudadanos y las redes, hecho del que se derivan cambios de estrategias en las industrias culturales. Los explicamos con más detenimiento en el artículo Las redes sociales y entretenimiento. Una apuesta hacia el verdadero ocio peligroso (Berlanga, 2011). En cuanto a la Persuasión, como botón de muestra, las redes sociales son un excelente instrumento para que productores y consumidores dialoguen y conozcan de una forma más cercana a sus clientes potenciales, fomentando así una comunicación fluida.

\subsection{Presencia de la Retórica en la comunicación de Internet}

Por tanto, un nuevo campo de estudio en la Red es la presencia y el papel de la Retórica. Si ya George Landow (1997: 120-121) reflexionó sobre el papel y las implicaciones de la Retórica antigua en el hipertexto y analizó los cinco componentes de la Retórica en sus aspectos constructivos y pragmáticos, Tomás Albaladejo va más lejos a la hora de tender puentes entre esta antigua disciplina e Internet. Según Albaladejo (2005) la elaboración de la una web responde a un proceso productivo retórico en cuanto a la organización semiótica del objeto significativo que construye; y así, con la inventio se obtiene y prepara los contenidos; con la dispositio, se estructura; con la elocutio lo expresa haciendo uso de todos los medios con los que cuenta, y con la actio o pronuntiatio lo comunica.

También Jerónimo Alayón (2009), siguiendo a Albaladejo abunda en las operaciones retóricas constituyentes de discurso en el hipertexto. El análisis que realiza este autor nos parece muy completo y acertado por lo que resumiremos la traslación que lleva a cabo de cada una de las operaciones retóricas, completándola con la opinión de otros autores y con la nuestra propia.

Brenda Estúpiñan (2009) argumenta que el diseño de interfaces responde a un proceso retórico en el que se realizan las operaciones de inventio, dispositio, argumentatio y actio con el fin lograr un producto persuasivo. En la misma línea, Alejandro Tapia (2003) recoge los elementos de la retórica clásica en el diseño de una web como un recurso orientador en la interpretación que el usuario realiza de la misma.

Otro autor que reseña la presencia de la Retórica en Internet es Javier Díaz Noci, en este caso, articulándola con el ciberperiodismo. En su obra Definición teórica de las características del ciberperiodismo: elementos de la comunicación digital (2008), emplea un criterio de clasificación de los cibertextos periódisticos al hilo de las partes del discurso retórico clásico. También en este artículo realiza un recorrido entre los autores que relacionan el discurso hipermedial con las figuras estilísticas o retóricas. 


\section{Criterios de clasificación retórica de los cibertextos periodísticos}

$\begin{array}{ll}\text { Topoi } & \text { Partes del discurso } \\ \text { Géneros narrativos } & \text { Inventio: Multilinealidad y poliacroasis } \\ \text { Géneros interpretativos } & \text { Dispositio: Estructuras hipertextuales } \\ \text { Géneros dialógicos } & \text { Actio: Interactividad } \\ \text { Géneros argumentativos } & \text { Elocutio: Recursos multimedia } \\ & \text { Memoria }\end{array}$

Imagen 1. Clasificación retórica de los cibertextos periodísticos. Fuente: Díaz Noci, 2008: 60

Por su parte, Francisco García García (2005) considera que las Tecnologías de la Información y la Comunicación, con sus características de no linealidad, hipertextualidad, interactividad y acción cooperativa, entre otras han vuelto a pone de moda a la Retórica. Para este experto en Comunicación Audiovisual y Retórica una primera prueba del poder expresivo de los recursos retóricos puestos al servicio de la técnica y de la ciencia es el uso de figuras, muy especialmente metáforas, para definir algunas operaciones de Internet, como puede ser "navegación" o "autopistas de la información".

En la misma línea, Roberto Gamonal realiza un sugerente análisis de los términos metafóricos en la Web, comenzando por la gran metáfora de la Red de Redes: "millones de puntos de comunicación esparcidos por la faz de la Tierra conectados por una intrincada red tejida de cables que ni la mitológica Aracne hubiera sido capaz de hilar mejor" (2004: web). También García (2007) se pregunta si existe una Retórica específica de Internet. Y concluye que, así como existen unos principios generales que explican la naturaleza retórica en todo tipo de discurso, asimismo existe una Retórica ligada a la propia naturaleza y grandes paradigmas de Internet: las bases de datos y su gestión del conocimiento, la hipertextualidad, la convergencia textual, la interactividad y la interactuación, la comunicación sincrónica y asincrónica y la accesibilidad universal.

\subsection{Presencia de la Retórica en las redes sociales}

Todos estos principios se aplican igualmente a las redes sociales que, por ser sitios web encaminados a facilitar una comunicación persuasiva, son en sí ya retóricos. Pero si hemos establecido esta relación atendiendo a la configuración de la interfaz de estas plataformas, queremos destacar que la unión de estos dos escenarios tiene su razón original en la naturaleza del discurso que se origina en las redes, discurso que ha recibido diferentes nombres según los estudiosos del tema. El más usado es Computer Mediated Communication o CMC (Baron, 2000: 158) lo que en español correspondería a comunicación vía Internet, electrónica, virtual o cibernauta. El origen de esta denominación se remonta al estudio de Hiltz y Turoff (1978) sobre comunicación humana 
mediante ordenador. Desde entonces ha sido el objeto de estudio de la llamada "etnografía virtual" (Hine, 2004). Lo cierto es que la convergencia tecnológica ha transformado no solo los códigos y lenguajes de la comunicación sino el fenómeno mismo de la comunicación y las prácticas e interacciones sociales asignadas a él.

Para comprender mejor este cambio de paradigma es preciso reparar en los rasgos explicativos de la comunicación en red. Un discurso que comparte rasgos tanto del discurso oral como del escrito; una comunicación interpersonal, que recoge el carácter eminentemente persuasivo del uso conversacional del lenguaje; en definitiva, una comunicación mediada por soportes tecnológicos, condiciones todas que exigen una revisión crítica de los modelos al uso.

Con todo, la Retórica en las redes sociales es un campo virgen que no ha producido aun trabajos científicos que directamente avalen la estrecha unión de estas dos realidades. Nosotros publicamos algunos artículos al respecto (Berlanga, 2010, Berlanga et. al, 2011) y con la investigación que resumimos a continuación profundizaremos en el entrelazamiento de la Retórica y nuestro presente comunicativo. Un presente mediado por el boom de las redes como nuevo paradigma.

\subsection{Análisis retórico de una red: el caso Facebook}

La red en la que nos centramos es Facebook, creación revolucionaria en el ámbito comunicativo. Actualmente es la herramienta de comunicación más utilizada y la red social que mayor número de seguidores abarca y cuyas cifras de registrados son incalculables ya que aumenta cada segundo. Según el informe de ONTSI (2011) Facebook es "la red social" y se ha convertido en sinónimo del concepto.

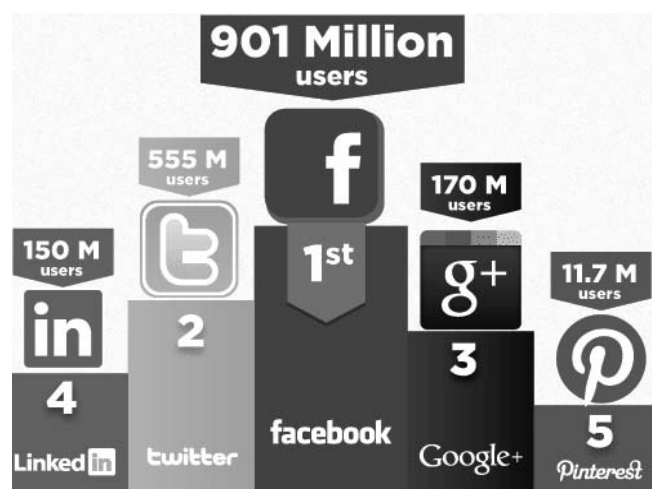

Imagen 3. Número de usuarios en las redes sociales más populares, a julio de 2012. Fuente: http://www.arturogoga.com/2012/05/21/comparacion-de-usuariosentre-las-redes-sociales-mas-populares-infografia/social-networking-users/

Recogemos los resultados de una investigación que hemos llevado a cabo sobre el lenguaje en los medios digitales, concretamente sobre los principios de Retórica clásica en las redes sociales, presentando como modelo el caso Facebook (Berlanga, 2012). En el proceso de investigación se analizó el discurso de la propia plataforma y 
el discurso que emplean los usuarios de la red y en sus relaciones entre sí. La metodología aplicada combinó las técnicas del análisis documental, el análisis de contenido de los muros de distintas microrredes y las entrevistas científicas a expertos. El trabajo de campo analizó 16 microrredes de Facebook, de perfiles con una edad comprendida entre los 16 y los 72 años. El total de usuarios que intervinieron fueron 200.

Como conclusiones principales se recogen las siguientes:

Es un hecho comprobado la presencia de la Retórica en la interfaz y en el discurso de los usuarios en la red, específicamente en Facebook.

La elaboración de la interfaz de Facebook responde a un proceso retórico que incluye invenio, dispositio, elocutio, memoria y actio.

La interfaz gráfica del usuario puede ser considerada como un nuevo texto retórico.

El discurso de los usuarios de las redes sociales está pleno de figuras retóricas.

Las figuras retóricas que se emplean en las redes sociales generan pensamiento, diálogo, comunicación más eficaz.

Sírvanos de ejemplo el muro de un usuario universitario. Este usuario posee una de las redes más densas retóricamente hablando: interviene 14 veces durante los 15 días de estudio, utilizando 56 figuras retóricas, 26 distintas entre sí, lo que supone una media de 4 figuras por intervención. A su vez, las 13 intervenciones de sus contactos recogen 34 usos de figuras, 15 diferentes entre sí. De esta forma la red presenta una media de 3,3 figuras por intervención, lo que la sitúa en el segundo puesto de densidad entre las redes analizadas.

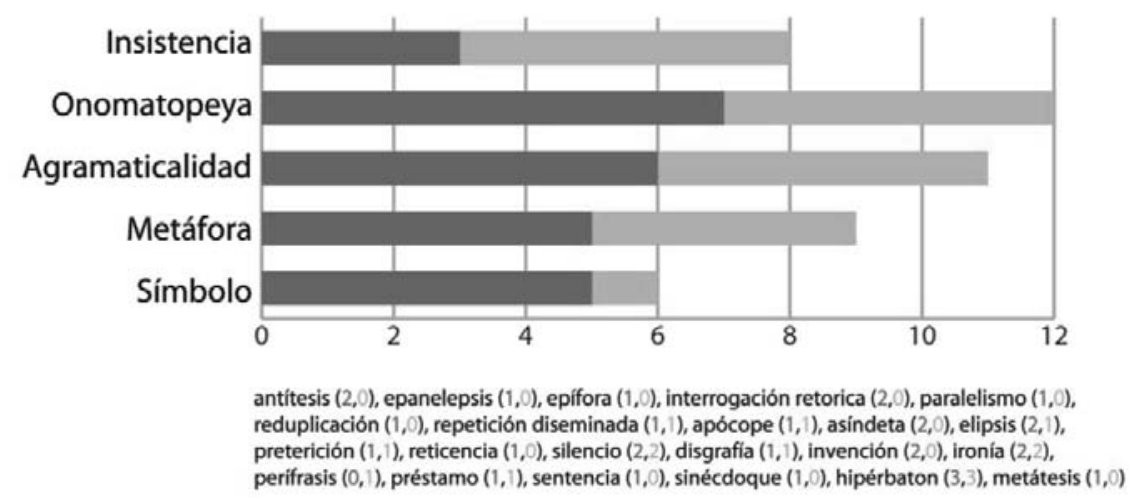

Imagen 3. Ejemplo gráfico del uso de figuras retóricas en las conversaciones del muro de usuario de Facebook de un alumno universitario de $1^{\circ}$ curso. Fuente: elaboración propia.

No obstante, ni la participación en Facebook ni la diversidad de uso de figuras son elevadas, dato que concuerda bien con el perfil adolescente del usuario titular.

En el usuario (color verde) hay un predominio de figuras de adición sobresaliendo entre ellas el uso de la onomatopeya (7). Otras figuras de este tipo son: la insistencia (3), la antítesis (2), la interrogación retórica (2) apanalepsis (1), epífora (1), paralelismo (1), y repetición diseminada (1). Entre las figuras de adición sobresale la me- 
táfora (5) y el símbolo (5) junto a invención (2), ironía (2), préstamo (1), sentencia (1) y sinécdoque (1). Entre las de supresión cobra peso la agramaticalidad (6) frente a asíndeton (2), elipsis (2) y silencio (2), apócope (1), preterición (1) y reticencia (1). Encontramos también 3 usos de hipérbaton y 1 metátesis.

Entre los ocho usuarios que aparecen en la red (color naranja) hallamos también un predominio de la insistencia, onomatopeya y agramaticalidad (5 usos respectivamente), 4 metáforas, 3 hipérbaton, 2 silencios, 2 ironías, y 1 uso de apócope, preterición, repetición diseminada, paráfrasis, préstamo y símbolo.

Pero además de los datos que nos ha aportado este ejemplo, la media de 2,7 figuras por intervención en el conjunto de los usuarios analizados, el uso de figuras en todos los perfiles y la variedad de las mismas demuestran sobradamente esta afirmación.

\section{Conclusiones. Hacia una docencia más... original}

1. Las nuevas formas de comunicación (redes sociales) y la teoría de la Retórica se aportan mutuamente los medios idóneos para lograr procesos comunicativos más eficaces y creativos. En el primer de los casos, la aportación de la Retórica a las redes sociales, se pueden concretar en: a) Recursos y estrategias para lograr una comunicación más eficaz; b) Un enorme valor añadido; una semiótica connotativa; c) Un espacio público que permite comunicarse sin trabas ni censura, una manera más de que el pueblo haga oír su voz y llegue a otras instituciones donde no tiene acceso, donde el debate es sesgado, donde no tienes un representante, sino que tú mismo dices y defiendes lo que piensas (el ágora del siglo XXI); d) Un método creativo para la generación de discursos en las redes sociales que puede ser muy útil para los profesionales de la comunicación. Si se estudia y se practica, una ayuda a que las relaciones personales y de los personajes que cada cual expone ante los demás hagan más vivible y digna la vida cívica, en asuntos públicos como la política y la ética; e) Pistas sobre cómo se organiza la información se presenta y se procesa en el marco de la comunicación por Internet.

En el caso de las aportaciones que las nuevas formas de comunicación hacen a una teoría de la Retórica se puede concretar la hipertextualidad, la ampliación del concepto de texto y unas nuevas formas de entender el texto, el discurso y la comunicación que se establece a través de ellos; en definitiva, un terreno de estudio, análisis y posibles soluciones.

2. proponemos el aprovechamiento de las redes sociales para formar en la ciencia Retórica. Ya que es una realidad comprobada la presencia de la Retórica en los discursos que emplean los usuarios, aunque sea un uso de forma inconsciente, sería interesante la creación de contenidos para aplicaciones que traten esta disciplina de una manera sistemática, lúdica y creativa.

3. Por tanto, como conclusión principal aportamos la necesaria inclusión de la disciplina retórica en el plan de estudios de los grados relacionados con la Comunicación. Nos unimos a David Pujante en su defensa categórica de la conveniencia de hacer un hueco a la Retórica en la enseñanza actual (Pujante, 2002: 140). Así se lograrían concluye este experto- poner en valor las potencialidades que esta vieja disciplina desplegaba; por un lado, construir el discurso de la verdad de un tiempo y un espacio 
determinados, esto es, el discurso de lo que era más útil para una sociedad dada; por otro, ofrecer los mecanismos de un auténtico servicio social: la capacidad para desmontar discursos falsos, equívocos, inapropiados o contraproducentes. Y es que:

"Si el relativismo cultural, nacido de las ideologías extenuadas, nacido cuando poderes mediocres, que pueden destruirlo todo, no saben convencer nos muestra la cara más terrible de la retórica (publicidad, economía, política); la retórica es, sin embargo, principalmente signo de la superación de la amoral razón, de la entelequia científica, de la necesidad de encontrarnos con un lenguaje de la calle, un lenguaje del día a día, de nuestro presente, el único tiempo con que contamos y en el que se insertan todos nuestros problemas. Cuando este planteamiento sea guía de la enseñanza, se habrá asentado la verdadera democracia en el corazón del hombre" (Pujante, 1999: 116-117).

Mirando a nuestro campo de estudio, en un momento histórico como el que vivimos, caracterizado por el éxtasis de la Comunicación, y a la vez por tal trepidación, inmediatez y superávit de información, que nos aboca, aun sin quererlo, a la despersonalización y la superficialidad, será de gran utilidad recuperar unos parámetros que devuelvan el pensamiento profundo y crítico en favor de una comunicación más humana. Las propuestas concretas de proyectos docentes, quedan pendiente para otra investigación.

\section{Referencias bibliográficas}

ALAYÓN, Jerónimo (2009): "Retórica y Discurso Hipertextual: Del trovador oral al trovador hipermedial. Notas para un estudio". Biblioteca Virtual Miguel de Cervantes: http://www.cervantesvirtual.com/obra-visor/retrica-y-discursohipertextualdel-trovador-oral-al-trovador-hipermedial-notas-para-unestudio-0/ $\mathrm{html} / 02401312$ 82b2-11df-acc7-002185ce6064_2.html\#I_0_. [fecha de consulta: 24 de julio de 2012].

ALBALADEJO, Tomás (2005): "La comunicación retórica en los sitios webs", en GARRIDO, Fernando (coord.): Actas del 20 Congreso On line del Observatorio para la Cibersociedad. Barcelona, Diputación de Barcelona, pp. 7-33.

ALBALADEJO, Tomás (2008): "Textualidad y comunicación: persistencia y renovación del sistema retórico" (La rethorica recepta como base de la retórica moderna). Revista Rhetorike: http://www.rhetorike.ubi.pt/00/pdf/albaladejo textualidad_comunicacion.pdf. [fecha de consulta: 24 de julio de 2012].

ANECA (2000): Libro Blanco Títulos de grado en Comunicación. Agencia Nacional de Evaluación de la calidad y la Acreditación, Madrid.

BARON, Naomi (2000): Alphabet to email. London, Routledge.

BERLANGA, Inmaculada (2010): "Ciberlenguaje y principios de retórica clásica. Redes sociales”.Enl@ce Revista Venezolana de Informacion, Tecnologia y Conocimiento, $\mathrm{n}^{\circ}$ 7. Zulia (Venezuela), Universidad de Zulia, pp. 47-61. 
BERLANGA, Inmaculada (2011): "Redes sociales y entretenimiento. Una apuesta hacia el verdadero ocio peligroso". Actas del II Congreso Internacional La sociedad digital. Icono 14, $\mathrm{n}^{\circ}$ 8: http://www.icono14.es/ocs/index.php?conference=2csd \&schedConf=-sociedaddigital $2 \&$ page=paper\&op=view\&path[]=704\&path[]=133. [fecha de consulta: 23 de julio de 2012].

BERLANGA, Inmaculada (2012): El lenguaje en los medio digitales. Principios de retórica clásica en las redes sociales. El caso Facebook. Granada, Universidad de Granada.

BERLANGA, Inmaculada "et al". (2011): "Facebook, nuevo ágora en un contexto de posmodernidad". I Actas del Congreso Internacional, Comunicación y Educación. Estrategias de alfabetización mediática. Barcelona, Universidad Autónoma de Barcelona.

DÍAZ NOCI, Javier (2008): "Definición teórica de las características del ciberperiodismo: elementos de la comunicación digital". Doxa Comunicación n ${ }^{\circ} 6$. CEU, Madrid, pp. 53-91.

GAMONAL, Roberto (2004): "La Retórica en Internet". Icono 14, 3: http://www.icono14.net/revista/num3/art1/all.html. [fecha de consulta: 6 de septiembre de 2012].

GARCÍA GARCÍA, Francisco (2005): “Una aproximación a la historia de la Retórica". Icono 14, 5: http://www.icono14.net/revista/num5/articulo1.htm. [fecha de consulta: 30 de agosto de 2012].

GARCÍA GARCÍA, Francisco (2007): "Una retórica de la publicidad: de la naturaleza inventiva a la verdad metafórica". Pensar la publicidad: revista internacional de investigaciones publicitarias, 2, 1: http://revistas.ucm.es/index.php/PEPU/article/view/PEPU0707220167A/15692. [fecha de consulta: 31 de julio de 2012].

HILTZ, Starr Roxanne \& Turoff, Murray (1993): The network nation: Human Communication via computer. Cambridge Massachussets, The MIT Press.

HINE, Christine (2004). Etnografia virtual. Barcelona, UOC.

LANDOW, George, P. (1997): "Wittgenstein, Genette y la Narrativa del lector" en LANDOW, George, P. (coord.): Teoría del hipertexto. Barcelona, Paidós.

ONTSI (2011). Estudio sobre el conocimiento y uso de las redes sociales en España: http://www.ontsi.red.es/ontsi/es/estudiosinformes. [fecha de consulta: 20 de julio de 2012].

PUJANTE, David (2002): "El lugar de la retórica en la enseñanza actual". Logo. Revista de Retórica y Teoría de la Comunicación 2: http://hdl.handle.net/2183/650. [fecha de consulta: 24 de julio de 2012].

SALAVERRÍA, Ramón (2012): Los periodistas obligados a renovarse, en Comunicando: http://blogs.unir.net/comunicacion/2012/06/12/los-periodistas-obligadosa-renovarse/. [fecha de consulta: 23 de julio de 2012].

TUBELLA, Inma \& ALBERICH, Jordi (2012): Comprender los media en la sociedad de la información. Barcelona, UOC. 


\section{Inmaculada BERLANGA FERNÁNDEZ}

Inmaculada.berlanga@unir.net

Paseo de Castellana 163, 8 28046 - Madrid

Universidad Internacional de la Rioja

Profesora Agregada de la Facultad de Empresa y Comunicación. UNIR

\section{Jordi ALBERICH PASCUAL}

jalberich@ugr.es

Facultad de Comunicación y Documentación

Campus Universitario de la Cartuja, s/n, 18071 - Granada

Universidad de Granada

Profesor Titular de Universidad. UGR 